\title{
Cu-Rich Precursors Improve Kesterite Solar Cells
}

\author{
Marina Mousel, * Torsten Schwarz, Rabie Djemour, Thomas P. Weiss, Jan Sendler, \\ João C. Malaquias, Alex Redinger,* Oana Cojocaru-Mirédin, Pyuck-Pa Choi, \\ and Susanne Siebentritt**
}

$\mathrm{Cu}_{2} \mathrm{ZnSn}(\mathrm{S}, \mathrm{Se})_{4}$ (CZTS(e)) absorbers for photovoltaic applications gain increasing interest in thin film solar cell research due to the abundance of the raw materials and have already achieved $11.1 \%$ efficiency with mixed sulfide-selenide absorbers. ${ }^{[1]}$ We concentrate here on pure selenide absorbers (CZTSe), since their open circuit voltage loss with respect to the bandgap is less than in sulfur-containing absorbers. ${ }^{[2]}$ Recently, CZTSe based solar cells have achieved a record efficiency of $9.15 \%,{ }^{[3]}$ where the absorber of this record cell was prepared by a high-temperature co-evaporation process without a further annealing step. Similar to $\mathrm{Cu}(\mathrm{In}, \mathrm{Ga}) \mathrm{Se}_{2}$, these CZTSe absorbers are grown with an intermediate $\mathrm{Cu}$-rich $(\mathrm{Cu} /(\mathrm{Zn}+\mathrm{Sn})>1)$ step, i.e., during the high temperature growth process the absorber composition has an excess of $\mathrm{Cu}$ during a limited time interval. The final absorber composition is $\mathrm{Cu}$-poor $(\mathrm{Cu})$ $(\mathrm{Zn}+\mathrm{Sn})<1)$. A similar Cu-rich growth step in $\mathrm{Cu}(\mathrm{In}, \mathrm{Ga}) \mathrm{Se}_{2}$ is beneficial for the solar cells: it leads to larger grains and it reduces the recombination activity. ${ }^{[3,4]}$ In the current work, we have prepared kesterite absorbers by a precursor-annealing process. This allows a very easy approach to include a $\mathrm{Cu}$ rich step by using a Cu-rich precursor. Precursors are grown at low temperature $\left(320^{\circ} \mathrm{C}\right)$ by co-evaporation with constant rates and annealed afterwards at high temperature $\left(500{ }^{\circ} \mathrm{C}\right)$ in an SnSe and Se atmosphere. ${ }^{[5]}$ The low deposition temperature of the precursor and the annealing atmosphere suppress the decomposition reaction of kesterite, which was observed at high temperatures. ${ }^{[6]}$ The potentially beneficial Cu-rich step is achieved during the co-evaporation step, i.e., the composition of the precursors after deposition is Cu-rich. In the following we show that untreated $\mathrm{Cu}$-rich precursors lead to a $\mathrm{Cu}-\mathrm{Sn}$-Se phase at the surface of the absorbers after annealing, which completely inhibits any solar cell performance. This $\mathrm{Cu}-\mathrm{Sn}-\mathrm{Se}$ phase develops from a $\mathrm{Cu}_{1.82}$ Se phase on the surface of the $\mathrm{Cu}$ rich precursors. Since the $\mathrm{Cu}$ selenide phase can be removed by etching in cyanide before annealing, absorbers made from etched $\mathrm{Cu}$-rich precursors do not suffer from a $\mathrm{Cu}$-Sn-Se phase

M. Mousel, R. Djemour, T. P. Weiss, J. Sendler, J. C. Malaquias, Dr. A. Redinger, Prof. S. Siebentritt University of Luxembourg

Laboratory for photovoltaics

41, rue du Brill, L-4422, Belvaux, Luxembourg

E-mail:marina.mousel@uni.lu; alex.redinger@uni.lu; susanne.siebentritt@uni.lu

T. Schwarz, Dr. O. Cojocaru-Mirédin, Dr. P.-P. Choi

Max-Planck-Institut für Eisenforschung

Max-Planck-Str. 1, 40237, Düsseldorf, Germany

DOI: 10.1002/aenm.201300543 at the surface. We will demonstrate in the following that they in fact show better performance than absorbers made from originally Cu-poor precursors.

Figure 1a shows the compositions of absorbers grown under $\mathrm{Cu}$-rich conditions, as determined by energy dispersive X-ray spectroscopy (EDX). A table of the measured EDX values is given in the Supporting Information. Both precursors and annealed samples are plotted in the phase diagram adapted from Dudchak et al. ${ }^{[7]}$ To plot the compositions in the phase diagram, Se is assumed to be stoichiometric. The precursors (black circles) are in fact Cu-rich. After annealing (grey circles) of these precursors, the composition shifts from the Cu-rich to the $\mathrm{Cu}$-poor side and ends up in the region of the phase diagram (Cu-poor and Zn-rich $(\mathrm{Zn} / \mathrm{Sn}>1)$ ) where the best solar cell efficiencies have been reported. ${ }^{[8,9]}$ This shift is due to addition of Sn during the annealing. Thus, although the precursor is Cu-rich the annealed absorber is $\mathrm{Cu}$-poor. However, no working solar cells can be made from absorbers prepared by annealing untreated $\mathrm{Cu}$-rich precursors; their power conversion efficiency is zero. The reason can be understood when considering the nature of the $\mathrm{Cu}$-rich precursors and the annealing process. It is well known from Cu-rich $\mathrm{Cu}(\mathrm{In}, \mathrm{Ga}) \mathrm{Se}_{2}$ that a $\mathrm{Cu}_{1.82}$ Se phase is formed at the surface, which can be removed by a cyanide etch. ${ }^{[10]}$ Similarly, a $\mathrm{Cu}_{\mathrm{x}}$ Se phase has been observed during the Cu-rich step in kesterites. ${ }^{[3]}$ If a $\mathrm{Cu}_{1.82} \mathrm{Se}$ phase also forms on the surface of the Cu-rich precursor, the $\mathrm{Cu}$ selenide will be transformed into a $\mathrm{Cu}-\mathrm{Sn}$-Se phase during the Sn rich annealing step. We have shown previously that a $\mathrm{Cu}$-Sn-Se phase at the surface of the absorber is detrimental to the solar cell. ${ }^{[11]}$ Figure $1 \mathrm{~b}$ compares the secondary ion mass spectrometry (SIMS) profiles of a standard Cu-poor precursor and a $\mathrm{Cu}$-rich precursor. $\mathrm{Cu}$ is enriched at the $\mathrm{Cu}$-rich precursor's surface, whereas no $\mathrm{Cu}$ enrichment is detected in the $\mathrm{Cu}$-poor precursor. The X-ray diffraction (XRD) pattern of a $\mathrm{Cu}$-rich precursor in Figure 1c (red curve) shows the reflections of $\mathrm{Cu}_{1.82} \mathrm{Se}$, indicating that the $\mathrm{Cu}$ enrichment at the surface is due to a $\mathrm{Cu}$ selenide phase, very similar to the case of $\mathrm{Cu}(\mathrm{In}, \mathrm{Ga})$ $\mathrm{Se}_{2}$. After annealing, absorbers obtained from $\mathrm{Cu}$-poor precursors yield solar cells with efficiencies around $4 \%{ }^{[11]}$ whereas absorbers from Cu-rich precursor do not yield working solar cells at all. Hence, one may assume that absorbers obtained from the $\mathrm{Cu}$-poor and from the $\mathrm{Cu}$-rich precursor exhibit substantially different surface compositions. Detecting this difference in surface composition by SIMS is difficult since the surface layer might be thin and SIMS data can be influenced by matrix effects, surface roughness or oxide layers. Therefore, we have performed complementary atom probe tomography 
(a)

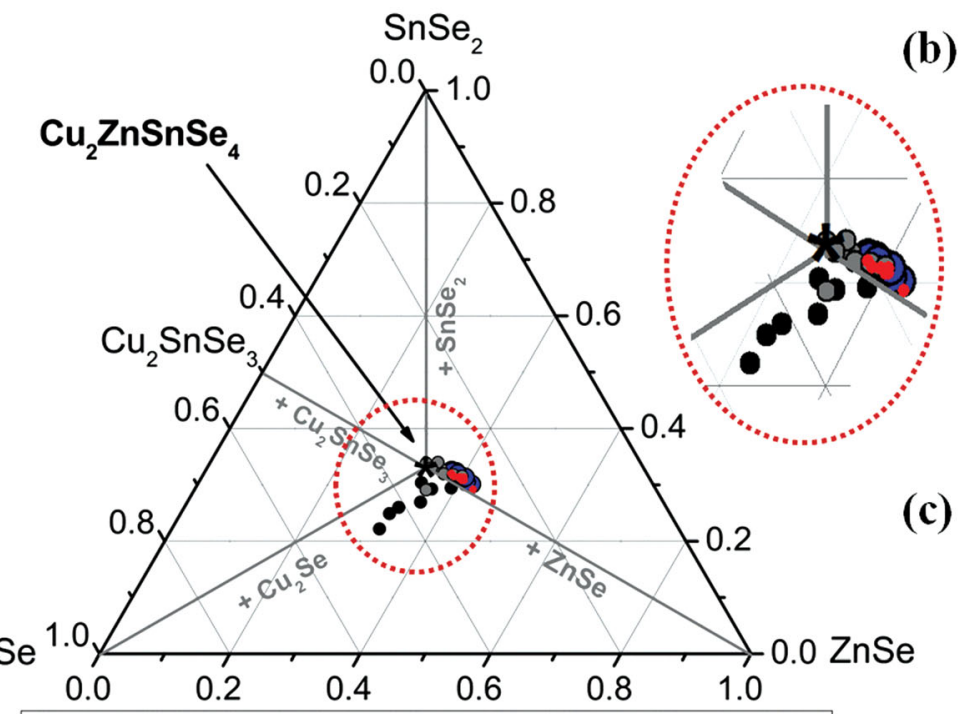

- Precursors (before annealing) before $\mathrm{KCN}$ etching

- Precursors (before annealing) after $\mathrm{KCN}$ etching

- Absorbers (after annealing) without $\mathrm{KCN}$ etching of the precursors - Absorbers (after annealing) with $\mathrm{KCN}$ etching of the precursors

(d)
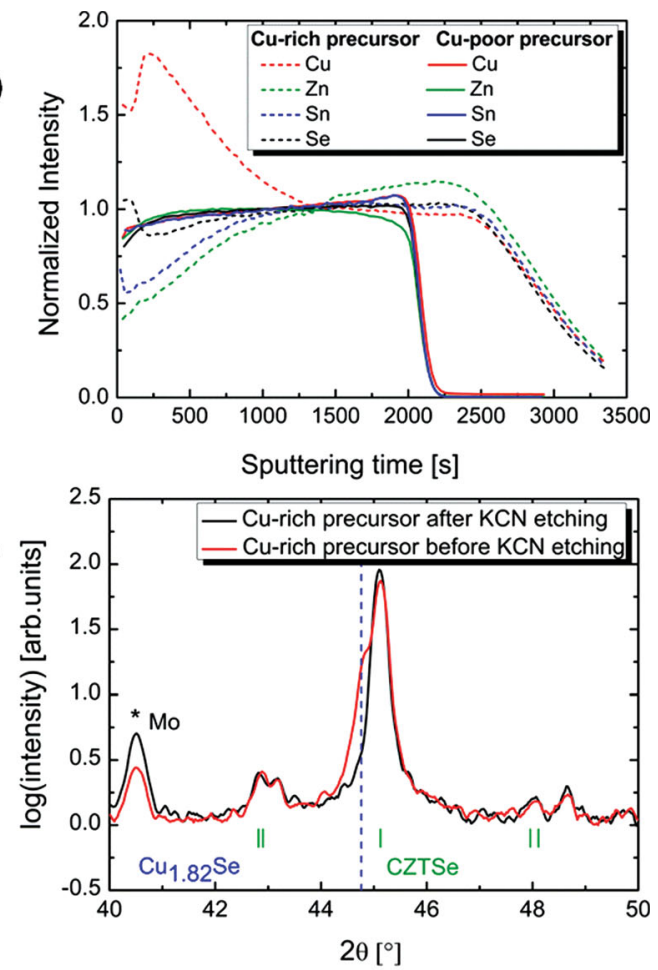

\begin{tabular}{|c|c|c|c|c|c|c|c|c|}
\hline Samples & $\eta \%$ & $\mathrm{~V}_{\text {oc }}[\mathrm{mV}]$ & $\mathrm{J}_{\mathrm{Sc}}\left[\mathrm{A} / \mathrm{cm}^{2}\right]$ & FF\% & $R_{s}\left[\Omega \mathrm{cm}^{2}\right]$ & $\mathrm{G}_{\mathrm{sh}}\left[\mathrm{mS} / \mathrm{cm}^{2}\right]$ & A & $\mathrm{J}_{0}\left[\mathrm{~A} / \mathrm{cm}^{2}\right]$ \\
\hline $\begin{array}{l}\text { Cu-rich precursors } \\
\text { With KCN etching (CA) }\end{array}$ & & & & & & & & \\
\hline Average & 6.0 & 348 & $3.09 * 10^{-2}$ & 56 & 0.48 & 5.3 & 2.20 & $7.6 * 10^{-5}$ \\
\hline $\begin{array}{l}\text { Cu-poor precursors } \\
\text { With KCN etching }\end{array}$ & & & & & & & & \\
\hline Average & 4.8 & 338 & $2.67 * 10^{-2}$ & 53 & 0.48 & 9.2 & 2.03 & $5.4 * 10^{-5}$ \\
\hline $\begin{array}{l}\text { Cu-poor precursors } \\
\text { Without KCN etching }\end{array}$ & & & & & & & & \\
\hline Average & 3.4 & 284 & $2.47 * 10^{-2}$ & 42 & 0.50 & 18.4 & 2.62 & $3.9 * 10^{-4}$ \\
\hline
\end{tabular}

Figure 1. a) Sample compositions plotted in the phase diagram adapted from ref. [7] as precursor without any surface treatment (black circles), precursors after KCN etching (red circles), after annealing without KCN etching (grey circles) and after annealing with KCN etching of the precursors before the annealing (blue circles). b) Normalized SIMS depth profile of a Cu-rich precursor, without etching, together with a standard Cu-poor precursor. c) X-ray diffractograms of a Cu-rich precursor before and after etching (the range from $25^{\circ}$ to $55^{\circ}$ is shown in the Supporting Information Figure S1). d) Solar cell parameters under illumination for $\mathrm{Cu}$-rich precursors using the CAPRI process, for $\mathrm{Cu}$-poor precursors with and without KCN etching before annealing.

(APT) studies. We observed different behavior for the differently prepared samples during the APT experiments. While the samples made from Cu-poor precursors showed high stability, APT tips extracted from the absorbers from $\mathrm{Cu}$-rich precursors fractured almost instantaneously at the beginning of the experiments. In one APT measurement it was possible to detect the $\mathrm{Cu}$-rich phase on the surface of the annealed $\mathrm{Cu}$-rich precursor. We measured a composition of $\mathrm{Cu}(34 \pm 2) \%$, Sn $(12 \pm 1) \%$, Se $(51 \pm 1) \%$, Zn $(4 \pm 0.5) \%$ for this phase, i.e., it is a Cu-Sn-Se phase which contains some $\mathrm{Zn}$. We conclude that this layer represents the detrimental phase. We note here that because of the small probed volume the detected composition determination is associated with a relatively large statistical error. Although absorbers made from $\mathrm{Cu}$-rich precursors may have favorable electronic properties, the $\mathrm{Cu}_{1.82} \mathrm{Se}$ layer on the precursor turns into an extremely detrimental layer with a very low $\mathrm{Zn}$ content during annealing in Se and Sn atmosphere and thus prevents solar cells made from $\mathrm{Cu}$-rich precursors by simple annealing.

Since it is likely that the detrimental phase has its origin in the $\mathrm{Cu}_{1.82} \mathrm{Se}$ phase on the surface of the precursor, the $\mathrm{Cu}$ selenide phase needs to be removed before annealing. It is well known that cyanides, such as KCN, are able to remove any $\mathrm{Cu}_{\mathrm{x}} \mathrm{Se}$ phase ${ }^{[10]}$ and thus the precursors were etched in KCN before annealing. The composition changes during the etching and annealing steps, as can be seen from Figure 1a. The first important observation is that the compositions shift from $\mathrm{Cu}$-rich to $\mathrm{Cu}$-poor directly after $\mathrm{KCN}$ etching, due to the removal of the $\mathrm{Cu}$ selenide layer (see EDX data available in the Supporting Information). Figure 1c shows XRD patterns of a Cu-rich precursor before and after etching, clearly indicating that the $\mathrm{Cu}_{1.82} \mathrm{Se}$ phase is eliminated by the etching process. The shoulders due to $\mathrm{Cu}_{1.82}$ Se reflexions are removed (a wider 
range from $25^{\circ}$ to $55^{\circ}$ can be seen in the Supporting Information Figure S1). Furthermore, the compositions of the etched precursors are in the range from which the best solar cells are produced and they do not shift anymore after annealing (see phase diagram in Figure 1a). Thus, the Cu-rich precursors lead to Cu-poor absorbers after annealing, independent of the treatment of the precursor. The improved precursor-annealing process, including an etching step, results in solar cells with a reproducible efficiency above $6 \%$ and is named CAPRI (from "cyanide absorber etching prior to annealing"). We assign the term CAPRI to absorbers made from Cu-rich precursors.

Solar cells prepared by the CAPRI process exhibit an efficiency which is considerably higher than the efficiency of solar cells obtained by the same annealing process from $\mathrm{Cu}$-poor and Zn-rich precursors. The power conversion efficiency of our best solar cell grown as precursor under Cu-poor conditions with no additional surface treatment, except for KCN etching before the buffer layer deposition, did not exceed $4.6 \%$, as was reported previously. ${ }^{[1]}$ The efficiency of solar cells issuing from the CAPRI process is higher without surface treatment after the annealing process (except for a short KCN etch just before the buffer deposition). The question arises if the improvement is due to the Cu-rich nature of the precursors in the CAPRI process or simply due to the etching of the precursors. Etching has been shown to be beneficial for finished chalcogenide absorbers, due to, for instance, cleaning the surface or increasing the interface band gap. ${ }^{[12,13]}$ Therefore, some $\mathrm{Cu}$ poor precursors were processed the same way as the $\mathrm{Cu}$-rich ones, by KCN etching prior to annealing. The table in Figure $1 \mathrm{~d}$ lists the average solar cell parameters for solar cells made from $\mathrm{Cu}$-rich precursors treated by KCN prior to annealing (CAPRI), from $\mathrm{Cu}$-poor precursors etched by KCN prior to annealing and from the same $\mathrm{Cu}$-poor precursors without $\mathrm{KCN}$ etching prior to annealing (the complete list of solar cells is given in Table S1 in the Supporting Information). Although the processing is the same, when etching $\mathrm{Cu}$-poor precursors prior to annealing, we would like to reserve the label CAPRI for Cu-rich precursors, since only they result in superior efficiencies, as discussed in the following. Comparing the Cu-poor precursors with and without etching, it is seen that etching of the precursors, in fact, does improve the solar cells by improving all photovoltaic parameters. Particularly the fill factor $(\mathrm{FF})$ and the open circuit voltage $\left(V_{\mathrm{OC}}\right)$ of solar cells made from etched $\mathrm{Cu}$-poor precursors are improved compared to unetched $\mathrm{Cu}$-poor precursors, which is consistent with the decrease of the reverse saturation current $J_{0}$ and of the diode factor A. Additionally, the series resistance $R_{\mathrm{S}}$ and shunt conductance $G_{\mathrm{sh}}$ decrease somewhat, contributing to an enhanced FF. The short circuit current $\left(U_{\mathrm{sc}}\right)$ increases slightly on average (about 10\% relative), but much less than the open circuit voltage, which increases about $20 \%$ relative on average. The increase in $V_{\mathrm{OC}}$ and the small change in $J_{\mathrm{sc}}$ indicate that the diode itself is improved by the etching process. We can only speculate that etching leads to a less defective interface with the CdS buffer or to improved properties of the space charge region. It can be concluded from this comparison, that etching before the annealing also improves Cu-poor precursors. Still, Cu-rich precursors after the CAPRI process do give better solar cells than Cu-poor precursors which are etched before annealing, as seen in the table in Figure 1d. Comparing the solar cell parameters from $\mathrm{Cu}$-rich and $\mathrm{Cu}$-poor precursors, both etched before annealing, the main advantage of cells made from Cu-rich precursors is a significant increase in short circuit current $J_{\text {sc. }}$ Open circuit voltage $V_{\mathrm{OC}}$ and fill factor FF also increase somewhat, but diode factor $A$, saturation current $J_{0}$, parasitic resistances and conductances do not change significantly. This observation indicates that the electronic properties of the diode do not change much by using Cu-rich precursors, but the collection properties are considerably improved. This observation can be explained by better transport properties of absorbers made from Cu-rich precursors. Figure 2a shows normalized quantum efficiency spectra (EQE) comparing several solar cells made from Cu-rich (red and black line) and Cu-poor (blue line) precursors, all etched before annealing. Although the solar cells show slightly different band gaps, which is attributed to different crystal modifications of the $\mathrm{Cu}_{2} \mathrm{ZnSnSe}_{4},{ }^{[14,15]}$ the main observation is that the current is improved for the $\mathrm{Cu}$-rich precursor in agreement with the observed short circuit currents. The improvement is mostly in the long-wavelength region, indicating a better collection near the back of the absorber and thus improved transport properties. A similar behavior is observed in chalcopyrite absorbers $\left(\mathrm{Cu}(\mathrm{InGa}) \mathrm{Se}_{2}\right)$, where etched Cu-rich absorbers exhibit better transport properties than Cu-poor ones. ${ }^{[16]}$

Solar cells prepared by the CAPRI process from $\mathrm{Cu}$-rich precursors have been analysed using temperature dependent $J-V$ measurements to study their dominant recombination path. ${ }^{[2]}$ Figure $2 \mathrm{~b}$ shows a comparison of the temperature dependence of the open circuit voltage of a solar cell made from a Cu-poor and a Cu-rich precursor, both etched before annealing. Since in both cases the open circuit voltage extrapolates to the band gap as determined by the quantum efficiency spectra (red and blue curve in Figure 2a), it is safe to conclude that these solar cells are not dominated by interface recombination. Nevertheless, they show a rather high reverse saturation current $J_{0}$ (table in Figure 1d) indicating a strong recombination channel which limits the efficiency of these solar cells. These limitations are also obvious from the open circuit voltages, which are, although improved, still about $650 \mathrm{mV}$ below the band gap value. Therefore, we studied in detail the absorbers prepared from etched $\mathrm{Cu}$-rich precursors. Room temperature confocal micro photoluminescence (micro PL) spectra were measured at different depths of the absorber (Figure 2c, all curves, besides the black one). Micro PL was measured at the surface after a $15 \mathrm{~s}$ etch in bromine methanol solution (grey curve in Figure 2c, labeled $15 \mathrm{~s})$ and in sputter craters with different depths, which are described by the different sputter times (labeled 150 to $2500 \mathrm{~s}$ ). The deepest sputter crater after $2500 \mathrm{~s}$ is close to the back of the absorber, where the absorber/Mo interface appears at about 3200 s. Most spectra show one or several emissions in the energy range between 0.9 and $1.0 \mathrm{eV}$. These are attributed to band-band recombination of several crystal modifications with slightly different band gaps, most likely stannite and kesterite phases. ${ }^{[14,15]}$ An additional transition is observed at $1.25 \mathrm{eV}$. This transition has been previously attributed to a defect related transition in ZnSe, due to resonant defect excitation. ${ }^{[17,18]}$ This $\mathrm{ZnSe}$ emission increases towards the back of the absorber. $\mathrm{ZnSe}$ has been previously related with the series resistance of the solar cells, ${ }^{[2]}$ however, using micro PL we are not able to 
(a)

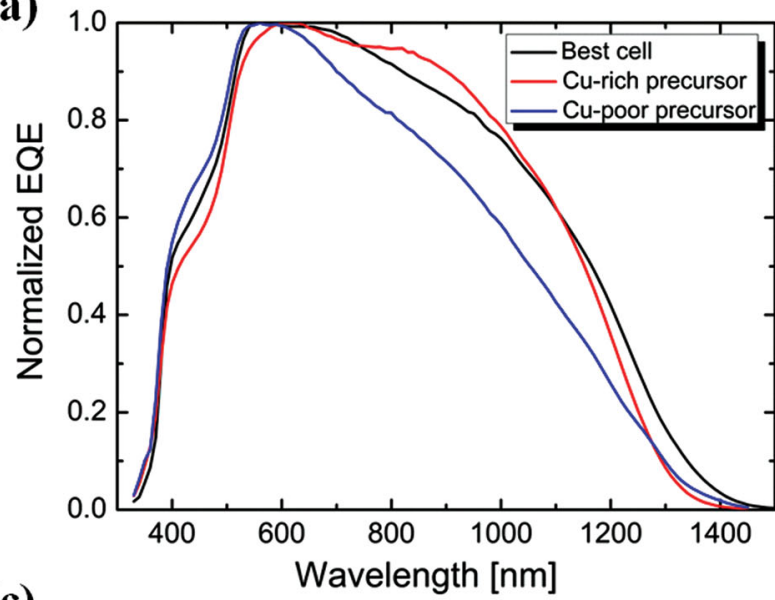

(c)

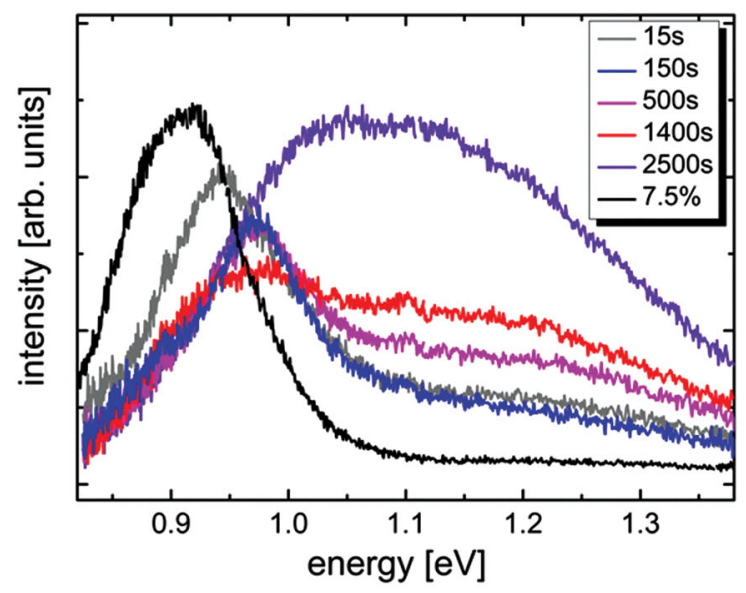

(b)

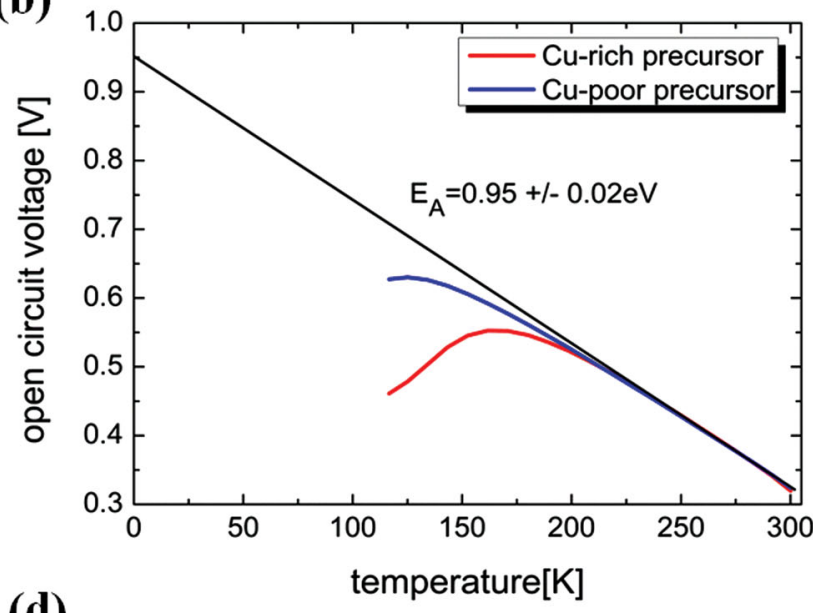

(d)

(e)
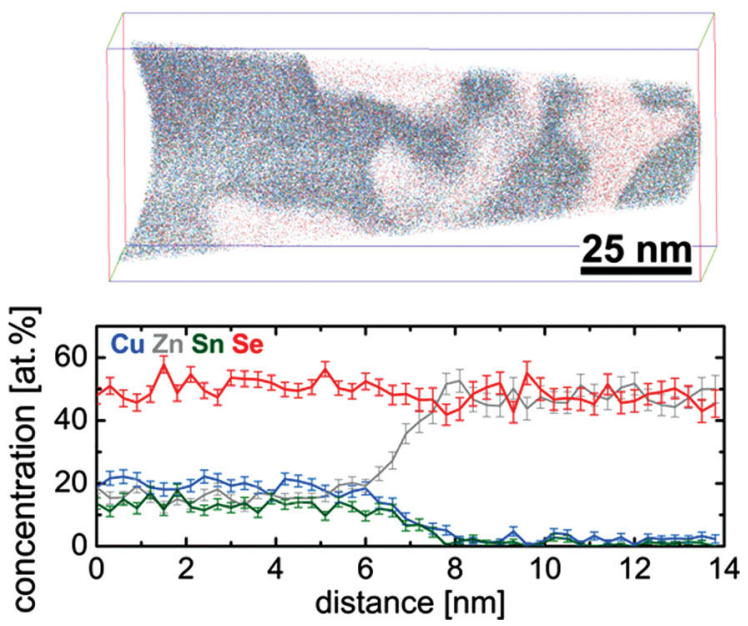

Figure 2. a) Normalized quantum efficiency spectra of solar cells made from etched precursors. The blue line originates from a Cu-poor precursor, the red and black line from Cu-rich precursors. b) Temperature dependence of the open circuit voltage for solar cells based on a Cu-rich precursor and on a Cu-poor precursor, both etched before annealing. c) Micro PL spectra measured at craters of different depths in the absorber, prepared by sputtering for the different times indicated. The grey line is not sputtered but after $15 \mathrm{~s}$ of etching in bromine methanol solution. The black line is the micro PL spectrum of the absorber, which resulted in the $7.5 \%$ cell. d) Atom probe dataset ( $6 \mathrm{~nm}$ thick slice) of CAPRI processed absorber based on a Cu-rich precursor (red dots indicate $\mathrm{Se}$, blue dots $\mathrm{Cu}$, grey dots $\mathrm{Zn}$ and green dots $\mathrm{Sn}$ ). e) Compositional line profile along the arrow indicated in Figure $2 \mathrm{~d}$.)

determine the exact location of ZnSe. Taking into account the excitation profile and additional carrier diffusion, the micro PL spectra shown in Figure 2c do not prove that ZnSe is present directly at the surface. However, they show that ZnSe is distributed across the absorber, with an increasing fraction towards the back contact. To further elucidate the distribution of $\mathrm{ZnSe}$, we have performed APT measurements on absorbers based on a $\mathrm{Cu}$-rich ${ }^{[19]}$ and a $\mathrm{Cu}$-poor precursor. Figure $2 \mathrm{~d}$ shows an APT map of an absorber based on a Cu-rich precursor after the CAPRI process. The analysed volume is located less than $200 \mathrm{~nm}$ away from the absorber surface. The absorber shows interconnected $\mathrm{ZnSe}$ regions of about $10 \mathrm{~nm}$ in size near the surface, extending further into the absorber. A compositional line profile across the interface between a $\mathrm{Cu}_{2} \mathrm{ZnSnSe}_{4}$ and a ZnSe region is shown in Figure 2e. The profile indicates that practically no $\mathrm{Cu}$ and $\mathrm{Sn}$ are present in the ZnSe region and that the $\mathrm{Cu}_{2} \mathrm{ZnSnSe}_{4}$ is very Cu-poor. This nanometer-sized network explains why ZnSe is detected throughout the whole absorber by micro PL. They can also explain why the series resistance is higher with more $\mathrm{Zn}$ at the surface ${ }^{[2]}$ because the current path between the ZnSe particles is limited in space and could also be subject to band bending. The micro PL spectra in Figure 2c indicate increasing ZnSe luminescence towards the back of the absorber. APT measurements near the back of the absorber also reveal a network of $\mathrm{ZnSe}$ inclusions. In fact, such ZnSe inclusions are not only observed in absorbers prepared by the CAPRI process but also in the absorber prepared by annealing an unetched Cu-poor precursor (see APT maps in the Supporting Information).

The strong recombination evident from the high saturation current can have a large number of causes. However, it is reasonable to assume that the interface between $\mathrm{Cu}_{2} \mathrm{ZnSnSe}_{4}$ and $\mathrm{ZnSe}$ contributes strongly to the recombination, since the interfacial area is huge and is very likely related with different doping levels of the two materials. That other causes can play a significant role as well, becomes clear when comparing the 
micro PL spectra and the saturation currents of a large number of solar cells. Cells exist which do not show the micro PL signature of $\mathrm{ZnSe}$ inclusions, but still have a saturation current above $10^{-4} \mathrm{~A} \mathrm{~cm}^{-2}$. However, all cells with a low saturation current (around $10^{-5} \mathrm{~A} \mathrm{~cm}^{-2}$ ) show no ZnSe signal in micro PL measurements. Therefore, the ZnSe inclusions are likely one cause among others for the high recombination. It can be assumed that the ZnSe inclusions can be reduced by increasing the temperature during the annealing process.

Therefore, additional solar cells have been prepared by annealing etched Cu-rich precursors with a slightly higher temperature by about $20{ }^{\circ} \mathrm{C}$. They result in an efficiency of $7.5 \%, 356 \mathrm{mV} V_{\mathrm{OC}}, 60 \% \mathrm{FF}$, and $35.4 \mathrm{~mA} \mathrm{~cm}^{-2} J_{\mathrm{SC}}$. The higher temperature leads mostly to additional improvements of the $J_{\mathrm{SC}}$, with small improvements of the $V_{\mathrm{OC}}$ and the FF. The QE spectrum is shown as the black curve in Figure 2a. It shows a slightly lower band gap than the two absorbers annealed at lower temperatures, which improves the current. Additionally, the spectrum shows the improved collection at long wavelength, typical of absorbers made from $\mathrm{Cu}$-rich precursors and indicative of better transport properties than in absorbers made from Cu-poor precursors. However, it is interesting to note that this cell shows a saturation current of only $1.5 \times 10^{-5} \mathrm{~A} \mathrm{~cm}^{-2}$. This is among the lowest saturation currents we have ever observed for $\mathrm{Cu}_{2} \mathrm{ZnSnSe}_{4}$ solar cells in our lab. The micro PL spectrum of this absorber after a short bromine methanol etch is shown in Figure $2 \mathrm{c}$ as the black line. There is no emission from ZnSe detectable. This supports our assumption that the ZnSe inclusions contribute to the recombination, although there can be many other causes of the high recombination. Also, at these higher temperatures we have compared $\mathrm{Cu}$-poor and $\mathrm{Cu}$-rich precursors, with the same results as shown for the lower temperatures: cells made from $\mathrm{Cu}$-rich precursors show better efficiencies.

In conclusion, we report on an improved precursorannealing process for kesterite solar cells, the so called CAPRI process, which includes an intermediate beneficial $\mathrm{Cu}$-rich step. The Cu-rich precursors show a $\mathrm{Cu}_{1.82}$ Se phase on the surface which, if not removed in a CAPRI process, is the starting point of the formation of a Cu-Sn-selenide related detrimental secondary phase. This phase formation can be suppressed by etching the $\mathrm{Cu}_{1.82}$ Se phase with KCN before annealing.

Our currently best solar cell prepared by this process has reached $7.5 \%$ power conversion efficiency for a pure selenide kesterite. The improved solar cell efficiency obtained with $\mathrm{Cu}$ rich precursors can be attributed to better current collection properties.

Direct evidence of nanosized ZnSe domains in the absorbers, likely responsible for the strong recombination in the bulk was given by atom probe tomography and photoluminescence.

\section{Experimental Section}

Polycrystalline CZTSe absorbers were produced by a precursorannealing process. Precursors with different compositions were obtained by co-evaporating all the elements ( $\mathrm{Cu}, \mathrm{Zn}, \mathrm{Sn}$ and $\mathrm{Se}$ ) at $320^{\circ} \mathrm{C}$ onto a molybdenum coated soda lime glass in a molecular beam epitaxy system. For a more complete description of the process see. ${ }^{[20]}$ The precursors were subsequently annealed for $30 \mathrm{~min}$ in a tube furnace at about $500{ }^{\circ} \mathrm{C}$ in an $\mathrm{H}_{2} / \mathrm{N}_{2}$ atmosphere of 1 mbar pressure. Elemental $\mathrm{Se}$ and solid SnSe were added to the annealing furnace to avoid decomposition of CZTSe during the heat treatment. ${ }^{[5]}$ The absorbers prepared with the new improved precursor-annealing process, the so called CAPRI process, were etched in $10 \mathrm{wt} \% \mathrm{KCN}$ for $1 \mathrm{~min}$ before annealing (CAPRI $\approx$ cyanide absorber etching prior to annealing). The morphology and the compositions before and after etching and after annealing were analyzed by scanning electron microscopy (SEM) and by $20 \mathrm{keV}$ energy dispersive X-ray spectroscopy (EDX). The EDX was calibrated by elemental or binary standards; the accuracy was about $1 \%$ in atomic composition. Secondary ion mass spectrometry (SIMS) depth profile measurements were performed on the absorbers with Cs+- ions. Since SIMS does not give absolute concentrations, the profile was normalized by setting the counts in the middle of the absorber thickness to 1. This allowed comparison of the relative changes between absorbers. Before solar cell finishing all the samples were etched in $5 \mathrm{wt} \% \mathrm{KCN}$ for $30 \mathrm{~s}$ in a standard procedure. After depositing the CdS buffer by chemical bath deposition, the window deposition was performed by magnetron sputtering of $\mathrm{i}-\mathrm{ZnO}$ and $\mathrm{Al}$-doped $\mathrm{ZnO}$. At last, a nickel-aluminum grid was deposited by e-beam evaporation for front contacting. The typical size for the solar cells is $0.5 \mathrm{~cm}^{2}$ total area. The current-voltage $(U-V)$ measurements were performed with a halogen lamp adjusted to $100 \mathrm{~W} \mathrm{~cm}^{-2}$. The current densities were calculated using the actual cell areas determined with the help of a camera under the microscope. The solar cell parameters were determined based on the method described by Sites et al. ${ }^{[21]}$ The micro PL measurements were performed on a home built confocal setup with an excitation laser-line of $514.5 \mathrm{~nm}$. The spectra were acquired at different depths by sputtering the absorber for different times followed by a short etching to remove the sputter damaged layer. This etching of the absorber was done for $15 \mathrm{~s}$ in a $0.02 \mathrm{M} \mathrm{Br} \mathrm{Me}-\mathrm{OH}$ solution. The craters of different depths were created by sputtering $\mathrm{Cs}^{+}$ions at $10 \mathrm{kV}$ acceleration. Atom probe tomography (APT) analyses of the final absorber were performed in pulsed laser mode, using a local electrode (LEAP 3000X HR, Cameca Instruments). The focused-ion-beam lift-out technique was used to prepare the samples (for more details see ref. [22]). A $5 \mathrm{keV}$ Ga beam was used for final APT tip sharpening in order to reduce beam damage due to $\mathrm{Ga}$ implantation.

\section{Supporting Information}

Supporting Information is available from the Wiley Online Library or from the author.

\section{Acknowledgements}

The authors would like to thank Masato Kurihara at TDK Corporation and Maxime Thevenin for prompt solar cell finishing, Thomas Schuler for comprehensive technical assistance and Nathalie Valle from the Centre de Recherche Gabriel Lippmann (CRP) for the SIMS measurements. Financial support by TDK Corporation and by the Luxembourgish Fonds National de la Recherche is gratefully acknowledged.

Received: May 16, 2013 Published online: September 19, 2013

[1] T. K. Todorov, J. Tang, S. Bag, O. Gunawan, T. Gokmen, Y. Zhu, D. B. Mitzi, Adv. Energy Mater. 2013, 3, 34.

[2] A. Redinger, M. Mousel, M. H. Wolter, N. Valle, S. Siebentritt, Thin Solid Films 2013, 535, 291.

[3] I. Repins, C. Beall, N. Vora, C. D. Hart, D. Kuciauskas, P. Dippo, B. To, J. Mann, W.-C. Hsu, A. Goodrich, R. Noufi, Sol. Energy Mater. Sol. Cells 2012, 101, 154. 
[4] J. Larsen, L. Gütay, S. Siebentritt, Appl. Phys. Lett. 2011, 98, 201910.

[5] A. Redinger, D. M. Berg, P. J. Dale, S. Siebentritt, J. Am. Chem. Soc. 2011, 133, 3320

[6] A. Weber, R. Mainz, H. W. Schock, J. Appl. Phys. 2010, 107, 013516.

[7] I. V. Dudchak, L. V. Piskach, J. Alloy. Compd. 2003, 351, 145.

[8] H. Katagiri, K. Jimbo, M. Tahara, H. Araki, K. Oishi, Mat. Res. Soc. Symp. Proc. 2009, 1165, 125.

[9] S. Siebentritt, Thin Solid Films 2013, 535, 1.

[10] Y. Hashimoto, N. Kohara, T. Negami, M. Nishitani, T. Wada, Jpn. J. Appl. Phys. Part 1 1996, 35, 4760.

[11] M. Mousel, A. Redinger, R. Djemour, M. Arasimowicz, N. Valle, P. Dale, S. Siebentritt, Thin Solid Films 2013, 535, 83.

[12] D. Regesch, L. Gütay, J. K. Larsen, V. Depredurand, D. Tanaka, Y. Aida, S. Siebentritt, Appl. Phys. Lett. 2012, 101, 112108

[13] M. Bär, B.-A. Schubert, B. Marsen, R. G. Wilks, S. Pookpanratana, M. Blum, S. Krause, T. Unold, W. Yang, L. Weinhardt, C. Heske, H.-W. Schock, Appl. Phys. Lett. 2012, 99, 222105.
[14] S. Siebentritt, S. Schorr, Prog. Photovoltaics 2012, 20, 512.

[15] R. Djemour, A. Redinger, M. Mousel, L. Gütay, S. Siebentritt, unpublished.

[16] S. Siebentritt, L. Gütay, D. Regesch, Y. Aida, V. Deprédurand, Sol. Energy Mater. Sol. Cells 2013, doi: 10.1016/j.solmat.2013.04.014.

[17] A. Redinger, D. M. Berg, P. J. Dale, R. Djemour, L. Gütay, T. Eisenbarth, N. Valle, S. Siebentritt, J. Photovoltaics 2011, 1, 200.

[18] R. Djemour, M. Mousel, A. Redinger, L. Gütay, A. Crossay, D. Colombara, P. Dale, S. Siebentritt, Appl. Phys. Lett. 2013, 102, 222108.

[19] T. Schwarz, O. Cojocaru-Mirédin, P. Choi, M. Mousel, A. Redinger, S. Siebentritt, D. Raabe, Appl. Phys. Lett. 2013, 102, 042101.

[20] A. Redinger, S. Siebentritt, Appl. Phys. Lett. 2010, 97, 092111.

[21] J. R. Sites, P. H. Mauk, Solar Cells 1989, 27, 411.

[22] K. Thompson, D. Lawrence, D. J. Larson, J. D. Olson, T. F. Kelly, B. Gorman, Ultramicroscopy 2007, 107, 131. 